We used the method of Bowen and colleagues (2009) to evaluate the feasibility of the SPAN+program in terms of acceptability, demand, implementation, practicality, integration, and possible efficacy. Qualitative data was collected through interviews and a focus group discussion with participating healthcare professionals. Quantitative data was collected through standardized questionnaires filled in by healthcare professionals and family caregivers at baseline and at four-month follow-up.

Results: Preliminary results show that the SPAN+ program supports healthcare professionals to increase attention for empowerment of residents living with dementia, by reflecting together with other healthcare professionals on what matters for each individual resident. Difficulties were reported in engaging family caregivers in the SPAN+ program.

Conclusion: The SPAN+ program seems valuable to increase the focus of healthcare professionals on a sense of identity, usefulness, control, and self-worth of people living with dementia in a nursing home, and to promote their empowerment.

\title{
416 - Family quality of life in Neurodegenerative Diseases and associated factors
}

\section{Eva González, Alba Aza, Isabel Vicario-Molina, María Gómez-Vela, Mạ Begoña Orgaz \& Marta Badia}

Introduction: Neurodegenerative diseases (NDs) are one of the major causes of dependency among older people. Since family members assume most of the care, the impact of NDs goes beyond the patient and affects the functioning of the entire family. Nonetheless, the concept of Family Quality of Life (FQOL) is still insufficiently developed in this field: the literature has focused on family caregivers from an individual perspective, paying less attention to the family unit. Hence, the objectives were to describe FQOL of people with NDs and to identify factors associated, from a holistic point of view.

Method: The sample consisted of 300 family members of patients with NDs (70\% females; mean age: 62.4) living in the cross-border region of Spain-Portugal, mostly in rural areas. The majority were primary caregivers. They completed the FQOLS-ND via telephone. This survey examined how the family perceived its FQOL at the global and domain-level, in terms of attainment and satisfaction (measured on a 5-point Likert scale). It also collected data on diverse respondents' and family characteristics.

Results: The average score in Global FQOL was 3.65 (SD = 0.70) for attainment and 3.69 (SD = 0.47) for satisfaction. By domains, the highest value was found in Family Relations and the lowest in Support from services. Twenty hierarchical multiple regressions examined the potential predictors of Global FQOL and the nine domains for attainment and satisfaction. Medium predictive values (from $R^{2}=.14$ to $R^{2}=.20$ ) were found in Financial wellbeing (satisfaction), Support from services, and Leisure. The number of perceived barriers to social-health services was a significant predictor in all the explanatory models (the most frequently cited being: long wait for service, services not available, problems with transportation, lack of information, and financial costs).

Conclusions: These results confirm that NDs are especially challenging in rural areas, where families feel more isolated and have fewer opportunities to receive professional support. Therefore, there is a need to design of a specific portfolio of services, resources and benefits that involves the key sectors of family welfare (public, private, third sector and family) and brings them closer to these areas, covering all the needs.

Keywords. Neurodegenerative disease; family quality of life; FQoLS-ND; Services. 